\title{
What causes my inflammatory bowel disease?
}

\author{
HUGH J FreEMAN, MD
}

F LLOWING THE 1865 DESCRIPTION of ulcerative colitis in the Union Army, this entity 'evolved' into a broad group of intestinal disorders characterized by an inflammatory process. Eventually, a number of more specific, even distinctive, entities emerged on the basis of clinical and pathological features as well as the more precise identification of different infectious agents. Today, ulcerative colitis is defined as an idiopathic disorder characterized by a combination of clinical, radiographic, endoscopic and histological features requiring exclusion of specific known etiological, particularly microbiological, agents. In 1960, this diagnostic problem in colitis became particularly accentuated by the recognition that Crohn's disease could involve the colon, sometimes as an exclusive clinicalentity. Since that time, considerable effort has been expended in defining these apparently different patterns of inflammatory bowel disease (IBD).

Ulcerative colitis is characterized by rectal bleeding, diarrhea, abdominal pain and weight loss. In the colon, the inflammatory process is characterized by diffuse change invaribly involving the rectum and extending proximally in the colon for variable distances. The small bowel is generally not involved. Crohn's disease, in contrast, is characterized clinically by fever, abdominal pain, diarrhea and weight loss. Bleeding may occur but is less frequent. The pathological features of these two diseases also contrast sharply. Changes in Crohn's disease tend to be characterized by focal ulcers or a segmental inflammatory process often granulomatous in nature. The small bowel and, indeed, any site within the gastrointestinal tract, may be involved indicating that Crohn's disease is a far more extensive disease than ulcerative colitis; the latter condition involves the colon alone. As a result of this emerging definition of IBD and the recognition of at least two probably distinctive entities - ulcerative colitis and Crohn's disease - concepts relevant to etiology have evolved. In particular, epidemiological, microbiological and immunological observations will be reviewed.

\section{EPIDEMIOLOGICAL CONSIDERATIONS}

Ulcerative colitis and Crohn's disease share many epidemiological and demographical features. Both entities are worldwide in distribution although both are much less commonly observed, recognized or reported from underdeveloped nations, particularly in the African and Asian continents. Except for northeast Scotland, the incidence of ulcerative colitis remains stable. In contrast, the incidence of Crohn's disease appears to be increasing, at least in most reported population studies from North America and Europe. In a recent report from northern Alberta, for example, the incidence figures reported for all patients with IBD rose dramatically from 1964 to 1981 - this rising incidence in northern Alberta was especially noteworthy for new patients with Crohn's disease. For most studies of Crohn's disease, younger age groups seem to be most significantly affected compared with elderly patients. In addition an increased incidence has been observed in urban rather than rural populations. Since the epidemiological expression of these two entities differs, it is unlikely that ulcerative colitis and Crohn's disease represent different 
pathological expressions in response to the same etiological factor. Although the small and large intestine likely have only a limited number of responses to different pathological stimuli, most believe that these epidemiological results reflect the likelihood that both disorders are distinct entities with some overlapping biological features.

Other epidemiological studies have examined factors that might play a more direct etiological role in IBD. Studies on migrant populations to British Columbia, for example, have provided some intriguing data. Both ulcerative colitis and Crohn's disease have rarely been reported in the Chinese population living in Hong Kong; however, following their emigration to Vancouver, the incidence of newly diagnosed ulcerative colitis and Crohn's disease were observed to have dramatically increased. These observations in a modern day migrant population seem to implicate directly some environmental factor, such as a dietary or microbiological agent, in the pathogenesis of these two diseases. A reduced intake of dietary fibre and excessive consumption of highly refined sugers or certain food additives have been considered. Excessive consumption of chemically processed fats (eg, margarine) have been implicated. In some countries, such as Australia and Sweden, the increased rates of use of margarine and other hydrogenated fats in production of shortenings, chocolates, cookies, wafers, corn flakes and other breakfast cereals have been associated with the rising incidence rates of Crohn's disease.

Other 'dietary factors' include alcohol and different pharmacological agents. Although studies on declared alcohol consumption have failed to define any specific relationship with IBD, a number of drugs have been implicated. These include oral contraceptives which have been associated with an inflammatory colonic disorder similar to Crohn's colitis. Studies in the United Kingdom and in the California community of Walnut Creek suggested that the incidence of ulcerative colitis and/or Crohn's disease increased in oral contraceptive users. This risk also seems to depend on the timing and duration of oral contraceptive use. Other studies, however, have failed to confirm this potential hazard.

Antimicrobial agents (including antibiotics) have also been implicated. Clostridium difficile has been observed to cause colitis per se, sometimes indistinguishable from either ulcerative colitis or Crohn's disease. In addition, C difficile may be superimposed on underlying colitis. In some patients, this organism has apparently played a significant role in development of toxic colitis and toxic megacolon. Other organisms, such as Yersinia enterocolitica, may also be involved in the pathogenesis of the disease following antibiotic administration. This agent has been associated with ileitis and/or colitis.

Another more recently recognized factor in epidemiological studies is the striking relationship of different IBD syndromes to cigarette consumption. In predisposed individuals, smoking may be an important determinant of the type of IBD. Active and passive smoking may both be important. A number of studies have now established a relationship between cessation of smoking and/or nonsmoking and the development or exacerbation of ulcerative colitis. In contrast, cigarette smoking is more common in patients with Crohn's disease. In some series, other contributing variables may be important. In a recent study from Alberta, for example, the largest proportion of patients were young urban females who actively smoked cigarettes or were constantly exposed to cigarette smoke, suggesting that smoking interacts with locale, sex and age of the patient to influence disease prevalence. The precise mechanism for these apparent effects of cigarette consumption require elucidation but it has been hypothesized that smoking results in an altered immunological response that, in the predisposed individual, may result in development of Crohn's disease rather than ulcerative colitis.

A number of social variables may also interact to produce an increased predisposition to development of IBD. Several factors, such as religious affiliation, have been considered. Studies conducted in Sweden, the Unitec Kingdom, United States and most recently, northern Alberta, have revealed that members of the Jewish faith have an increased risk for development of IBD, particularly Crohn's disease. In contrast, however, a high prevalence of IBD has not been observed in Israel. In Tel Aviv, immigrants to Israel from Europe and North America were observed to have a higher prevalence rate than the Israeli-born population. It has been hypothesized that the patient's genetic background, possibly coupled with the higher socioeconomic status and urban rather than rural environment, were more significant factors than religious affiliation.

A number of epidemiological and familial studies have also been conducted to determine if there are genetic factors that might play a role in predisposing individuals to development of IBD. Several studies have identified a high prevalence in relatives, particularly siblings, especially in Crohn's disease. At the same time, concurrent disease in married couples has been only rarely recorded. This implies that familial aggregation and disease clustering must largely be due to genetic rather than shared environmental factors. Multiple members of the same family may be observed in up to $20 \%$ of patients with ulcerative colitis and up to $40 \%$ of patients with Crohn's disease. In addition, there is a high degree of concordance in monozygotic twins. Ulcerative colitis is observed more frequently in family members if the proband has ulcerative colitis. Crohn's disease is observed more frequently in family members if the proband has Crohn's disease. Occasionally, in up to $20 \%$ of all patients, there is some familial overlap between the two conditions. It is believed that the development of IBD in relatives living in separate geographic areas is evidence supporting a primarily inherited etiological, rather than environmental, factor. IBD also frequently is seen in association with a number of other genetically-based conditions. For example, HLA-B27-associated ankylosing spondylitis may accompany IBD in the same patient or 
be observed with increased frequency in families with colitis. Genetic chromosomal disorders like the Hermansky-Pudlak and Turner's syndrome, may be associated with an increased incidence of IBD. Finally, recent studies indicate that apparently disease-free relatives of patients with IBD have altered permeability to foreign substances in the intestinal tract.

\section{MICROBIOLOGICAL CONSIDERATIONS}

Initially both Crohn's disease and ulcerative colitis were believed to be due to specific infections involving the small and/or large intestine. At the beginning of the 20th century, for example, intestinal tuberculosis was frequently suspected, but in most patients its presence could not be confirmed. Despite the suspicious appearance of the intestinal tract in these patients, the tuberculosis organism could not be detected in resected tissues. In retrospect, many of these patients likely had Crohn's disease. Similarly, ulcerative colitis has been confused with a clinical illness caused by specific infectious organisms, including salmonella, shigella and campylobacter. In recent years, a number of 'new' organisms have also been detected, related in part to the emergence of newer microbiological methods, particularly with selective bacterial growth media.

A particularly popular avenue of investigation in recent years has been the search for a transmissible agent, including a virus or specific bacterial organism. Evidence for a viral etiology, particularly in Crohn's disease, was suggested from animal transmission experiments, electron microscopy and studies showing cytopathic effects in tissue culture systems. In 1976, the hind foot pads of normal and immune-deficient mice were injected with tissue homogenates from ileal and mesenteric nodes of patients with Crohn's disease and resulted in granulomas - these could then be transmitted to the foot pads of other mice. Similar findings were observed in rabbits using ileal homogenates from patients with Crohn's disease; in one study, the histological changes of
Crohn's disease were observed in the ileum of the rabbits. Although later investigators were not able to reproduce consistently these results, first and second generation passage were reported in the mouse foot pad model. More recent animal transmission experiments resulted in the induction of B cell lymphoma in nu-nu mice strains with tissue homogenates from Crohn's disease. Although these results may have been caused by a virus, other possibilities have included an atypical mycobacteria, antigen-antibody complexes or a foreign body that might induce a granulomatous inflammatory reaction.

Initial electron microscopic studies also suggested the presence of viral particles in Crohn's disease tissues, but subsequent studies from different laboratories failed to confirm these observations. Similarly, investigations assessing the cytopathic effects of material derived from Crohn's disease patients in tissue cultures have also yielded contradictory results. In patients with ulcerative colitis or Crohn's disease, for example, the presence of cytomegalovirus was suggested by cytopathic effects in tissue cultures, indirect immunofluorescence, antibody tests and electron microscopy. However, serum titres for cytomegalovirus antibodies were no different from control patients and later cRNA-DNA hybridization studies failed to confirm the presence of cytomegalovirus in patients with IBD. It is thought now that this virus simply infects sites in the gastrointestinal tract that are already ulcerated rather than being the primary cause of ulceration; further research, however, is required.

Evidence for other viral infections have also been rigorously investigated. Materials from patients with Crohn's disease and ulcerative colitis, for example, have been inoculated into embryonic eggs and a variety of different cell lines in tissue cultures. Considerable interest in other viruses (including a picanoravirus and a rotavirus) have resulted, but extensive studies in other laboratories failed to show that these agents are relevant.

A number of bacterial agents have also been of particular interest in recent years. Some have been considered important since specific medications used to treat IBD may have antibacterial properties. For example, metronidazole, an antibiotic that is very effective against anaerobic bacteria, appears to be useful in selected patents with Crohn's disease. Similarly, mycobacteria have been extensively studied, especially with Crohn's disease. Interestingly, one of the drugs used in the treatment of IBD, 5-aminosalicylic acid (5-ASA), is very closely related pharmacologically to para-aminosalicylic acid, an agent used in the treatment of tuberculosis. Some studies have reported the presence of certain strains of mycobacteria in tissues cultured from patients with Crohn's disease. Research in this area currently is focused on a variety of candidate organisms and their products (endotoxins, hemolysins, neurotoxins). Much work needs to be done to determine their relevance, if any, to the etiology and/or pathogenesis of IBD.

\section{IMMUNOLOGICAL CONSIDERATIONS}

At present, no definite primary abnormality in the immune system or immune regulation has been detected in either ulcerative colitis or Crohn's disease. Very detailed studies of different aspects of humoral and cell-mediated immunity in IBD have been done but none has isolated a specific immune defect. It appears that most immunological phenomena that have been explored during periods of active disease and during periods of remission are secondary to the disease per se and are so-called 'epiphenomena'. Generalized malnutrition or specific nutrient deficiencies may also cause some secondary immunological changes. Nevertheless, immunological events are exceedingly important, if not causative, in producing many of the observed changes in the intestinal tract of patients with IBD; immune-mediated reactions may cause significant tissue damage in the intestinal tract contributing further, but only secondarily, to the inflammatory disease process.

Altered humoral immunity has been described in IBD. Increased serum antibodies to Escherichia coli somatic $\mathrm{O}$ anti- 
gens, colonic epithelial cell surface and other antigens (eg, parietal cells), as well as the presence of experimentallyinduced anticolon antibodies, may reflect the presence of mucosal injury and secondary alterations in mucosal permeability. A wide variety of 'inflammatory reactants' have been observed in IBD patients (eg, orosomucoid, haptoglobin, ceruloplasmin, transferrin, etc) but their relevance is unknown. Finally, some patients with IBD develop reduced reticuloendothelial function and vulnerability to infection; this may be clinically manifested by the presence of splenic hypofunction and atrophy.

A variety of complement alterations have been reported during active disease. The metabolism of certain complement components may be altered and the concentration of specific complement components may be increased or decreased. Defective responsiveness to specific chemotactic substances and development of antibodies to activated complement components have been described. Immune complex mediated injury may occur in IBD patients with either ulcerative colitis or Crohn's disease. These complexes cause activation of complement components, activation of lysosomal enzymes and release of other inflammatory mediators.

Cell-mediated immune responses may also be altered in either ulcerative colitis or Crohn's disease. Circulating T and $B$ lymphocyte populations are not substantially changed in most patients although, in some, responsiveness to specific mitogens may be altered. In part, this functional alteration may represent a nutritionally-mediated change. Cell-mediated immune responsiveness to specific antigens generally is normal in ulcerative colitis and Crohn's disease, although malnutrition may result in diminished responsiveness to common skin test antigens (eg, candida). Some circulating lymphocytes from patients with either ulcerative colitis or Crohn's disease may be cytotoxic for autologous colonic epithelial cells. Alterations in circulating lymphocyte populations, however, may not truly be relevant toalterations in intestinal lymphocyte populations. Thus, recent re- search efforts have focused on intestinal lymphocyte populations, their physiology as well as regulatory factors, and mechanisms involved in their function and cellular interaction with other cells, hormones, growth factors and inflammatory mediators. These studies have also focused on genetically-mediated immunoregulatory activity including immune response and immune suppressive genes.

The immune response also involves a number of other nonlymphocytic cell types including circulating leukocytes, particularly neutrophils. Altered neutrophil function has been only studied to a limited extent but changes have been described in both ulcerative colitis and Crohn's disease. Some changes, such as altered neutrophil metabolism and increased phagocytic activity, may be secondary and induced by active disease while altered chemotactic responsiveness may reflect the presence of other inflammatory mediators or inhibitors. Macrophage function may also be altered substantially in some patients with IBD. A great deal of research effort is still required to elucidate the pathogenetic effects and consequences of altered immune responses and immune regulation in IBD.

\section{CONCLUSIONS}

There remains considerable speculation and controversy regarding the nature and cause of ulcerative colitis and Crohn's disease. A specific set of clinical and pathological criteria permit separation of these two entities in most instances although there may well be overlap in about 10 to $15 \%$. It is possible that the intestinal tract has only a limited number of pathological responses to different causes or, alternatively, these two entities may represent different expressions of the same disease due to a single cause. There are some data to support this 'unitary concept' of IBD, ie, ulcerative colitis and Crohn's disease are variants of the same disease. Either condition may occur simultaneously in the same family, possibly even in the same patient, and it is recognized that there are a range of intestinal and extraintestinal features that can occur in both ulcerative colitis and Crohn's dis- ease. Alternatively, it may be that their occurrence in the same family may reflect common genetic factors while co-existence of both conditions in the same individual may reflect misinterpretation of clinical and pathological information. Some clinical features may be a secondary phenomena. Although still uncertain, most of the available evidence does support the view that ulcerative colit is and Crohn's disease are most likely two diseases with at least a partially differing and multifunctional pathogenesis. Several factors have recently been itemized as possible 'clues' to the etiology of either disease.

\section{Ulcerative colitis}

- is not a contagious disease and the existence of the usual type of transmissible agent is unlikely

- has a familial occurrence suggesting that one (possibly more) genetic factor is important and predisposes to the disease

- has a periodic clinical course with active and quiescent intervals, pos. sibly related to immunological factors

- by definition, involves the rectum and extends proximally within the colon for variable distances - this distribution suggests either that the colonic mucosa distally in the rectum substantially differs from more proximal colonic mucosa or, alternatively, the quantity or quality of some intraluminal factor changes within the colon

- by definition, involves only the colon and, at least initially, is limited to the mucosa rather than the deeper layers of the colonic wall - this may relate to the regional nature of the disorder, some specific characteristic of the colonic mucosa compared with mucosa at other sites in the gastrointestinal tract or, possibly, a luminal factor

\section{Crohn's disease}

- an increased familial incidence im. plies a genetic factor (as in ulcerative colitis) 
- a rising frequency in Crohn's disease has been recorded in recent years, particularly in modern migrant populations, implying at least one environmental factor, such as an infectious agent or dietary factor

- the inflammatory process may be far more extensive in Crohn's disease than in ulcerative colitis and involve any site from the mouth to the anus raising the possibility of a systemic inflammatory disease

- the presence of granulomas in the tissue, particularly in the intestinal tract and local lymphoid tissues (but not in ulcerative colitis), provides some evidence for insoluble antigen absorption

- localization of the disease process is segmental in Crohn's disease, rather than diffuse as in ulcerative colitis, with 'skipped regions' of normal intestine suggesting that access to tissues by the initiating event substan- tially differs from that observed in ulcerative colitis

- there is a predilection for the ileocecal area rather than the rectum as in ulcerative colitis and the tendency for recurrent disease after resection in the 'neo'-terminal ileum may be due to the rich lymphoreticular system in this region of the intestinal tract

The cause(s) of ulcerative colitis and Crohn's disease are unknown. Evidence, however, has accumulated to support the hypothesis that a precipitating event occurs leading to an initial acute inflammatory process that later becomes chronic and perpetuating, particularly in a genetically predisposed individual. Some environmental agent, possibly a viral or bacterial agent, a bacterial toxin or a specific food product or chemical, may induce an inflammatory process in the susceptible individual mediated by a complex series of immunological reactions.

Much of the present research activity is focused on defining this initiating event in the inflammatory process as well as its activation and modulation by a wide variety of inflammatory mediators involved in this inflammatory process. If the initiating events or factors that control and regulate this process could be better understood, more specific therapies might be devised that could modulate and/or dampen the destructive immunological effects that result. Identification of specific genetic, environmental and infectious factors that play either an etiological or pathogenetic role in these disorders requires elucidation. Finally, new pharmacological therapies that might alter this inflammatory process are being explored. 


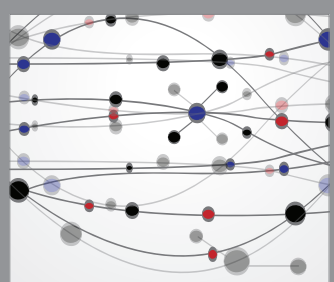

The Scientific World Journal
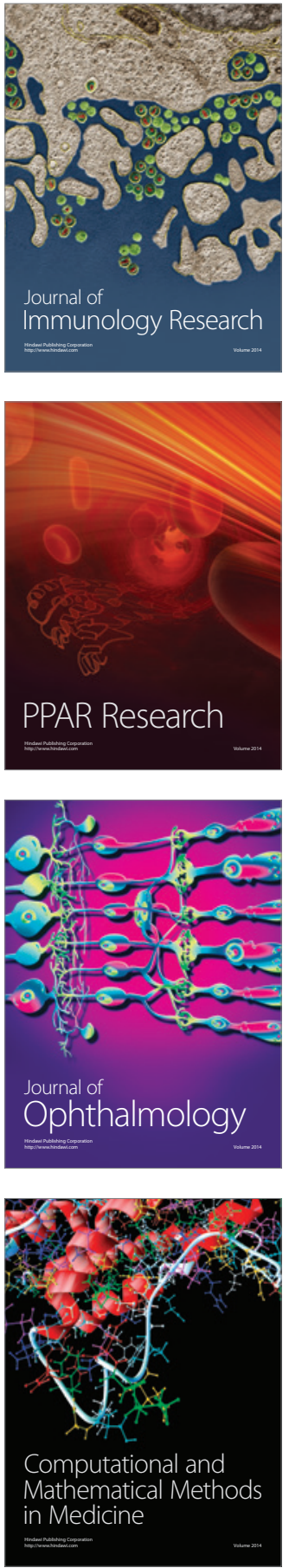

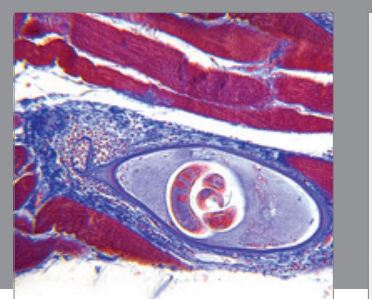

Gastroenterology Research and Practice

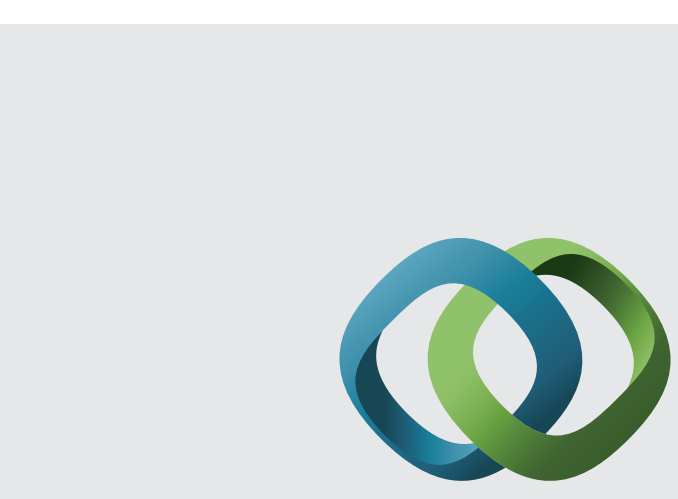

\section{Hindawi}

Submit your manuscripts at

http://www.hindawi.com
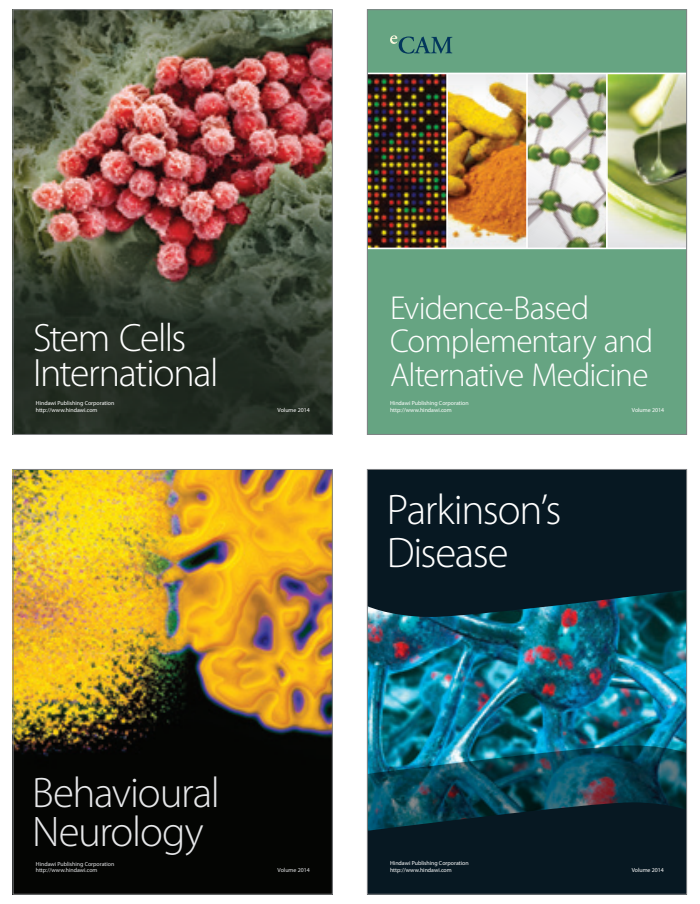
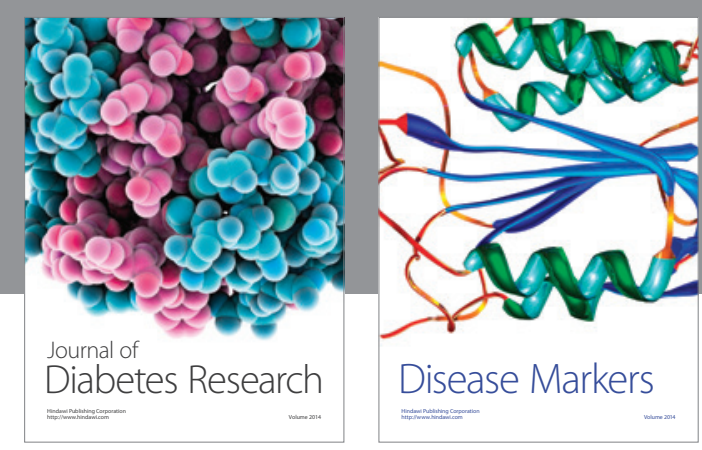

Disease Markers
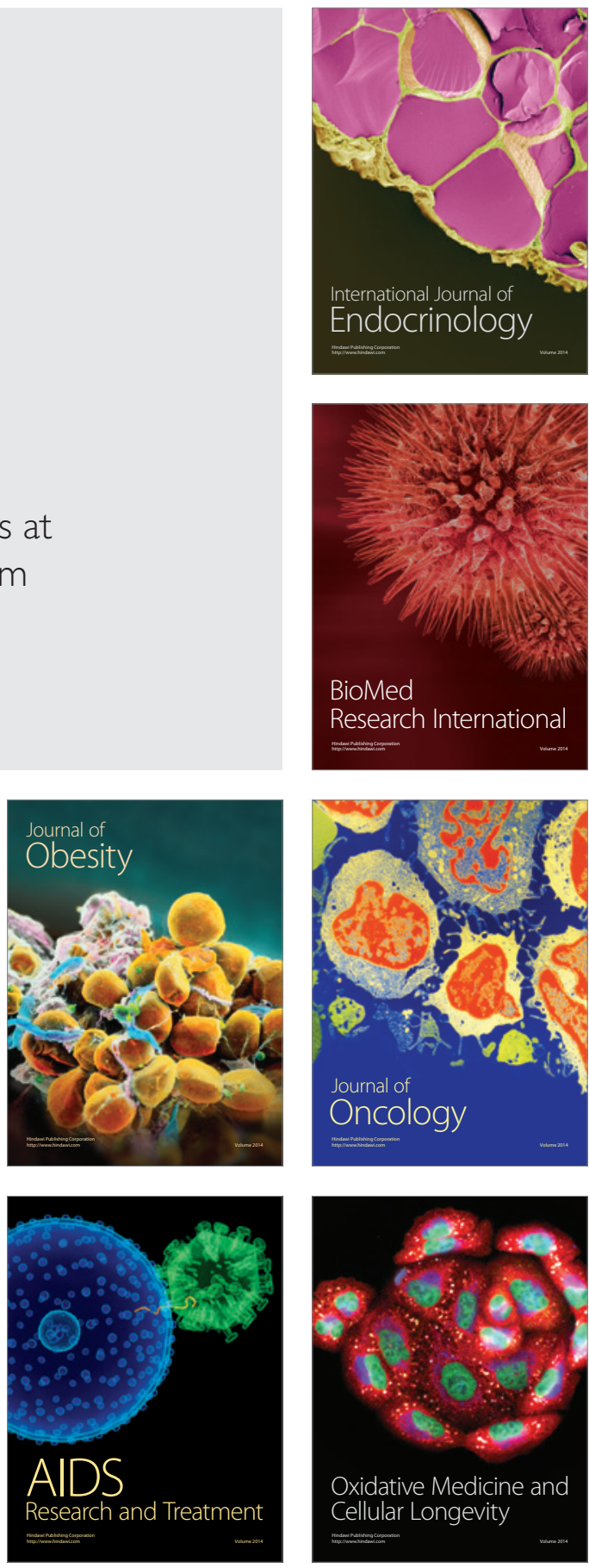